\title{
Prospects for combining immune checkpoint blockade with PARP inhibition
}

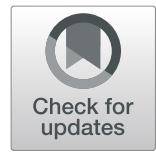

\author{
Anping $\mathrm{Li}^{1+}$, Ming $\mathrm{Yi}^{2+}$, Shuang Qin², Qian $\mathrm{Chu}^{2}$, Suxia $\mathrm{Luo}^{1 *}$ and Kongming $\mathrm{Wu}^{1,2^{*}}$ (D)
}

\begin{abstract}
The immunogenicity of a cancer cell is derived from accumulated somatic mutations. However, on the contrary to increased immunogenicity, anti-cancer immune response tends to be feeble. This impaired anti-cancer immunity could be attributed to multiple factors including loss of immunodominant epitopes, downregulation of major histocompatibility complex, and immunosuppressive microenvironment, as well as aberrant negative co-stimulatory signals. Immune checkpoint inhibitors block negative co-stimulatory signals such as programmed cell death-1 and cytotoxic T-lymphocyte-associated protein 4, ultimately reactivating anti-cancer immunity. Immune checkpoint inhibitors elicit potent anti-cancer effect and have been approved for multiple cancers. Nevertheless, there still are significant potential improvements for the applications of checkpoint inhibitor, especially considering frequent resistance. Recent studies demonstrated that additional PARP inhibition could alleviate resistance and enhance efficacy of immune checkpoint blockade therapy via promoting cross-presentation and modifying immune microenvironment. We proposed that PARP inhibitors could enhance the priming and tumor-killing activities of T cell, boost the whole cancer-immunity cycle, and thereby improve the response to immune checkpoint blockade. In this review, we focused the latest understanding of the effect of PARP inhibitors on anti-cancer immunity and PARP inhibitors combining immune checkpoint blockade therapy. Moreover, we summarized the preclinical and clinical evidence and discussed the feasibility of this combination therapy in future clinical practice.
\end{abstract}

Keywords: PARP inhibitor, DNA damage response, PD-1, PD-L1, CTLA-4, Immunotherapy, Combination therapy, Tumor immune microenvironment

\section{Background}

Cancer cells harbor substantial gene mutations and possess abnormal protein expression pattern. According to the specificity of expression, aberrantly generated proteins could be classified as tumor-associated antigens (TAAs) and tumor-specific antigens (also called neoantigens) [1-3]. TAAs refer to proteins remarkably overexpressed on cancer cells compared with normal cells. Neoantigens are proteins exclusively expressed on cancer cells due to mutationmediated sequence alterations $[4,5]$. TAAs and neoantigens determine cancer immunogenicity and initiate the cancer-immunity cycle [6]. Although host immunity could theoretically recognize tumor-derived materials and retard tumor growth, a subset of cancer cells escape from immune surveillance and develop into visible tumor lesions [7].

\footnotetext{
* Correspondence: luosxrm@163.com; wukm_lab@163.com

${ }^{+}$Anping Li and Ming Yi contributed equally to this work.

'Department of Medical Oncology, The Affiliated Cancer Hospital of

Zhengzhou University \& Henan Cancer Hospital, Zhengzhou 450008, China Full list of author information is available at the end of the article
}

An effective anti-cancer immune response relies on the robust cascade reaction including release and presentation of cancer antigens, priming and activation of $\mathrm{T}$ cells, trafficking and infiltration of $\mathrm{T}$ cells, and recognizing and killing tumor cells [8]. However, one or more steps of this cancer-immunity cascade reaction are undermined in cancer patients. A growing body of evidence indicated that loss of cancer-specific immunodominant epitopes and $\mathrm{T}$ cell repertoire, downregulation of antigen processing, and presentation machinery, as well as immunosuppressive microenvironment could lead to immune tolerance to tumor antigens $[9,10]$. Immunotherapy restores or enhances anti-cancer immune response via eliminating inhibitory immune components, transferring additional tumor-specific $\mathrm{T}$ cell clones, reshaping immunosupportive microenvironment [11-14]. Immune checkpoint inhibitor (ICI), cancer vaccine, and adoptive $\mathrm{T}$ cell transfer have been applied in multiple cancers [15-18]. Nevertheless, due to the spatial 
heterogeneity and dynamically evolving cancer antigen spectrum, it is hard to cure a tumor by monotherapy and tumors cells eventually acquire resistance [19]. Therefore, immunotherapy-based combination strategy attracts extensive attention for synergistic efficacy and lower risk of resistance [20, 21].

Poly ADP-ribose polymerase (PARP) inhibition induces synthetic lethal effect in cancer cells with a deficiency in homologous recombination (HR) [22]. Besides, PARP inhibitor (PARPi) could promote the priming of anti-cancer immune response and enhance Th1-skewing immunity, as well as modulate immune microenvironment [23]. The immunological effect of PARPi is multifaceted which might be favorable to boost cancer-immunity cycle and enhance the efficacy of ICI treatment [24]. This review focused on preclinical studies and clinical trials of PARPi combined with ICI therapy, as well as prospects and challenges of this combination therapy.

\section{PARP inhibition}

\section{The role of PARP in DNA damage response}

Genome intensity is challenged by continuous DNA damage events [25]. Normal cells could detect and repair DNA damages by multiple pathways: (1) DNA singlestrand break (SSB) repair pathways including base excision repair (BER), nucleotide excision repair (NER), and mismatch repair (MMR) and (2) DNA double-strand break (DSB) repair pathways such as HR and nonhomologous end joining (NHEJ) [26]. For cells harboring mutations in gene coding, the key components of DNA damage response (DDR) such as BRCA1/2, TP53, and $\mathrm{MSH} 2$, inadequate elimination of genome mutations increases the risk of carcinogenesis after DNA damage events [27]. Actually, cancer cells often possess inadequate repertoire of DNA damage repair pathways and highly depend on certain DNA repair pathways to avoid lethal DNA damages [27, 28].

PARP is a core DNA damage sensor in DDR, which binds to damaged DNA lesions, catalyzes the generation of negatively charged poly (ADP-ribose) chains, remodels the structures of damaged chromatin, and recruits DNA repair-related protein complex $[29,30]$. Then, PARP is dissociated from the DNA damage site by autoPARylation [31]. It has been well established that PARP mainly participates in BER-mediated SSB repair, as well as other multiple DDR pathways (Fig. 1a) [32].

\section{PARPi and synthetic lethal effect}

The anti-cancer effect of PARPi has not been completely understood yet, which is initially attributed to inhibition of catalytic effect [33]. As a result, SSB persists and eventually develops into replication-dependent DSB [34]. In normal cells, when both HR and NHEJ pathways are available in G2/M stage, HR pathway is preferentially adopted to repair DSB [35]. HR is an effective repair approach with high fidelity which uses the sister copy of damaged sites as the template [36]. However, for some cancer cells with HR deficiency such as BRCA1/2 mutations, NHEJ pathway is utilized for DSB repair [37]. NHEJ is an error-prone repair pathway with low fidelity which could induce unsustainable DNA damages (e.g., chromosomal rearrangements) and eventual cell death (Fig. 1b) [38]. Based on this synthetic lethal effect, numerous PARPis are developed including Veliparib, Rucaparib, Olaparib, Niraparib, and Talazoparib, which are mainly applied in cancer patients with BRCA1/2 mutations [39-44].

Then in clinical practices, some phenomena emerged which could not be fully explained by synthetic lethal theory. Firstly, the capability of PARPi to inhibit PARP catalytic activity is not closely correlated to its cellkilling ability in BRCA-mutated tumors [32]. Besides, PARPi could induce greater tumor-killing effect than PARP depletion [32]. Actually, these phenomena are attributed to the PARP trapping potency of PARPi. In general, all agents belonging to PARPi could interfere with the interaction between PARP and its cofactor ( $\beta$-nicotinamide adenine dinucleotide), inhibit PARylation activity, and trap PARP on damaged DNA chain [45]. Apart from uncontrolled DNA damage accumulation, DNAPARP complex-mediated cytotoxicity also contributed to tumor cell death [46]. Originally, the potent anti-cancer effect of PARPi was found in BRCA1/2 deficient ovarian cancer patients. Later, the clinical use was expanded to BRCA-mutated breast cancer, ovarian cancer, pancreatic cancer, prostate cancer [47-49]. Recently, it was found that some non-BRCA-mutated tumors shared therapeutic vulnerabilities with BRCA-mutated tumors [50]. These non-BRCA-mutated tumors termed as BRCAness tumors that often harbor other alterations in HR genes except for germline BRCA deleterious mutations [50]. Patients with BRCAness tumors could benefit from PARPi treatment as well [50].

In spite of the great success of PARPi in a particular population, there are some problems needing to be properly resolved, especially acquired PARPi resistance [51]. This resistance is primarily attributed to the inactivation of key molecules involved in NHEJ pathway, such as 53BP1, the loss of PARP, and secondary mutations in BRCA restoring the activity of HR [52-54]. Combination therapy is a feasible strategy to enhance efficacy and decrease treatment resistance. It is notable that PARPi combining chemotherapy is easy to induce dose-limiting toxicity [55]. In the meanwhile, based on the hypothesis that patients with mutations in BRCA1/2 or other HR components are prone to possess higher mutation burden, multiple clinical trials are ongoing to explore the efficacy of combination therapy of PARPi and immune checkpoint inhibitors [56]. The interim analysis of 


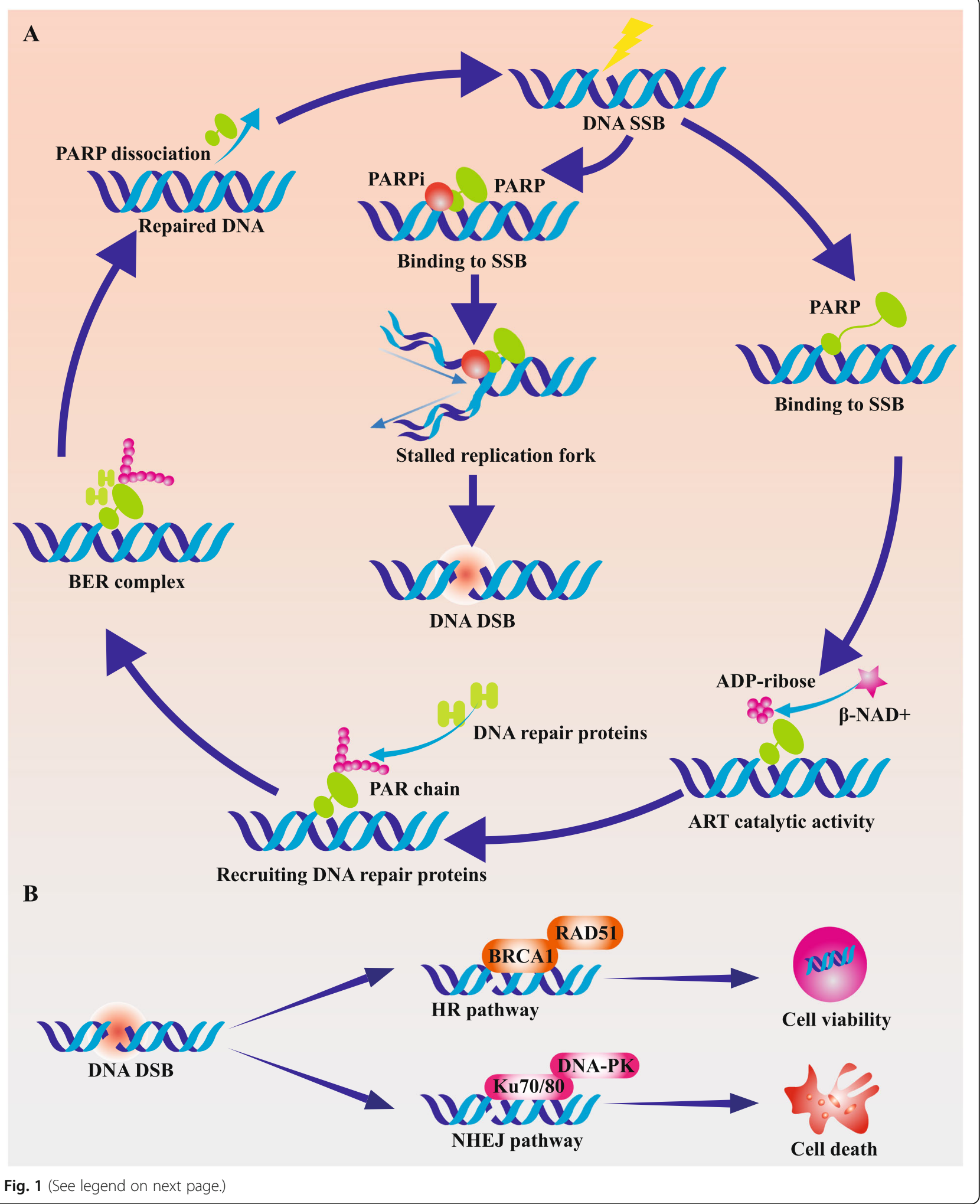

SOLO1 (NCT01844986) showed that BRCA1/2-mutated ovarian cancer patients could significantly benefit from Olaparib treatment (hazard ratio of cancer progression or patient death $=0.30,95 \%$ CI $0.23-0.41, P<0.001$ ) [57]. In another phase III study POLO (NCT02184195), 154 germline BRCA1/2 mutant pancreatic cancer patients 


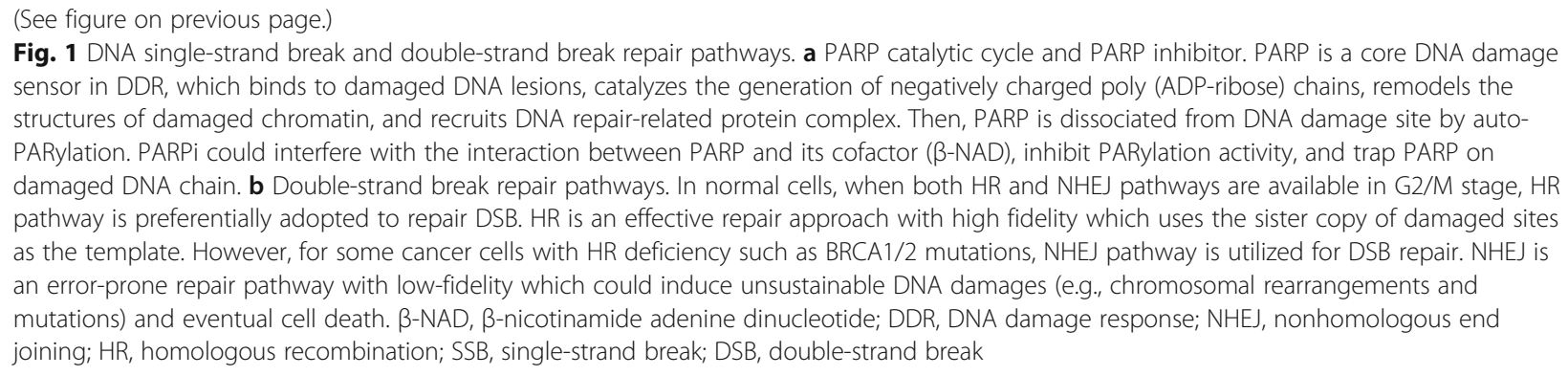

were enrolled [58]. The results of the POLO study indicated that patients undergoing Olaparib treatment tended to have a prolonged progression-free survival than the placebo group (median progression-free time: 7.4 months vs. 3.8 months, hazard ratio $=0.53,95 \% \mathrm{CI}$ $0.35-0.82, P=0.004)$ [58].

\section{Immune checkpoint inhibitor}

Two signals are required for the activation of naive $\mathrm{T}$ cells [59]. The first signal is the specific binding between $\mathrm{T}$ cell receptor (TCR) and antigenic peptide-major histocompatibility complex (pMHC) [59]. Contrary to the first signal, the second signal is a non-antigen-specific pathway which depends on the binding between costimulatory molecules and corresponding ligands [60]. The balance between positive and negative costimulatory signals is crucial for the activation and tolerance of $\mathrm{T}$ cells $[61,62]$. Among negative co-stimulatory molecules, programmed cell death-1 (PD-1) and cytotoxic T-lymphocyte-associated protein 4 (CTLA-4) are relatively well-studied signals which are also termed as immune checkpoints.

\section{PD-1/PD-L1 signaling pathway}

PD-1 is expressed on multiple activated immune cells including $\mathrm{T}$ cells, B cells, natural killer (NK) cells, and dendritic cells (DCs) [63]. As the main ligand of PD-1, PD-L1 is constitutively expressed on a wide variety of immune cells and non-immune cells [63, 64]. Besides, the expression of PD-L1 could be induced by inflammation response [65]. In the context of TCR stimulation, the immunoreceptor tyrosine-based inhibitory motif (ITIM) and immunoreceptor tyrosine-based switch motif (ITSM) of PD-1 are phosphorylated which further recruit SHP1/2 and counteract TCR/CD3-CD28 mediated tyrosine phosphorylation $[66,67]$. As a result, the downstream signaling cascade of TCR is inhibited by PD-1/ PD-L1 axis. Besides, PD-1/PD-L1 signal could also suppress intercellular PI3K-Akt and Ras-Raf-MAPK signaling pathways, which further downregulates glycolysis, the metabolism of amino acid and fatty acid oxidation, as well as cell proliferation [68, 69]. Dysregulated metabolism promotes the differentiation of $\mathrm{T}$ cells towards regulatory $\mathrm{T}$ cells (Tregs) [70].

\section{B7-CTLA-4 signaling pathway}

CTLA-4 is a negative co-stimulatory molecule which mainly regulates the priming and activation of $\mathrm{T}$ cells in peripheral lymphatic organs [6]. CTLA-4 is constitutively expressed on Tregs and transiently upregulated on conventional $\mathrm{T}$ cells after activation [71]. CTLA-4 competitively antagonizes CD28 by binding to CD80 (B7.1) and CD86 (B7.2) [72]. Subsequent internalization of CD80/CD86-CTLA-4 complex decreases the abundance of available co-stimulatory molecule ligands and elevates the threshold of $\mathrm{T}$ cell activation [73]. Besides, through intracellular ITIM, CTLA-4 could counteract TCR/CD3mediated tyrosine phosphorylation and inhibit the signal transduction of TCR [74].

\section{Clinical application of ICI}

Since the first ICI was approved for metastatic melanoma patients in 2011, numerous ICIs have entered clinical practice $[75,76]$. Anti-CTLA-4 and anti-PD-1/PD-L1 treatment exhibited a potent and durable tumor-killing effect in multiple advanced cancers such as triple-negative breast cancer, non-small lung cancer, renal cell cancer, melanoma, and urothelial bladder cancer [77-81]. However, the clinical application of ICIs is limited by low response rate [82]. Although a series of biomarkers have been adopted to predict the efficacy of ICI and select patients before treatment beginning, the actual primary and acquired drug resistance has not been completely overcome [17]. Some factors have been verified as the core determinants of the efficacy of ICIs treatment such as tumor mutation burden, MMR deficiency, the status of tumorinfiltrating lymphocytes (TILs), PD-L1 expression, and immunosuppressive microenvironment [83-86].

The development of ICI-based combination therapy provides a novel perspective to enhance ICI efficacy and overcome treatment resistance. ICIs are usually combined with chemotherapy, radiotherapy, and targeted therapy, as well as antiangiogenic therapy [20, 87-89]. Generally, the combination therapy is aiming to promote antigen presentation, broadening $\mathrm{T}$ cell repertoire, and 
impairing immunosuppressive components [90]. The results of NCT02763579 showed that atezolizumab plus cytotoxic chemotherapy (carboplatin and etoposide) showed more potent anti-cancer effect than chemotherapy in advanced small cell lung cancer patients (median overall survival 12.3 months vs. 10.3 months, hazard ratio $=0.70, \quad 95 \% \quad$ CI $0.54-0.91, \quad P=0.007 ; \quad$ median progression-free survival 5.2 months vs. 4.3 months, hazard ratio $=0.77,95 \%$ CI $0.62-0.96, P=0.02$ ) [15]. Besides, multiple clinical trials exploring other ICI-based combination strategies are ongoing.

\section{The rationale of PARPi combining ICI therapy}

\section{Tumor mutation burden and neoantigen}

The relationship between tumor mutation burden and efficacy of ICI has been confirmed in previous studies $[78,91]$. Tumor mutation burden is regarded as a surrogate of neoantigen burden which heralds the therapeutic response after ICI treatment [78]. Tumor mutation burden is closely related with DDR deficiency [92]. Hypermutated tumors often harbor one or more mutations in key components of DDR pathways such as hMSH2, BRCA1/2, and POLE [92-94]. After receiving anti-PD-1/ PD-L1 treatment, patients with DDR deficiencies had a higher response rate compared with patients without these deficiencies [95]. Thus, patients with HR or other DDR deficiencies might be candidates for both PARPi and ICI therapy.

PARPi-mediated catastrophic DNA damage is a favorable factor for ICI therapy, even though the influence of HR deficiency on tumor mutation burden is weaker than MMR deficiency [92]. After receiving PARPi treatment, accumulated chromosome rearrangements generate plenty of neoantigens and elevate the immunogenicity of tumor. Theoretically, PARPi could increase the sensitivity of patients to ICI therapy by increasing mutation burden.

\section{DNA damage and CGAS-STING pathway}

Apart from tumor mutation burden, DDR-mediated immune responses collaborate with ICI which remodel tumor immune microenvironment and boost the cancerimmunity cycle [96]. Due to the genomic instability and incomplete DNA repair repertoire, DNA damages accumulate and could not be fully repaired in tumor cells. As a result, these DNA damages persist in a low level which might increase the possibility of the exposure of doublestrand DNA (dsDNA) in cytoplasm [97]. Following the stimulation of cytoplasmic dsDNA, cyclic GMP-AMP synthase (cGAS) is activated and catalyzes the generation of cyclic-dinucleotide (CDN) [98]. CDN is a second messenger which promotes the conformational change of stimulator of interferon genes (STING) [99]. Active STING mainly initiates the downstream TBK1-
IRF3-Type I IFN pathway [100]. Besides, STING could activate NF-kB pathway which cooperates with IRF3 to upregulate the generation of type I IFN [101]. Type I IFN has a substantial influence on systemic immune response and regulates multiple components in anticancer immunity especially DCs, natural killer cells (NKs) and T cells (Fig. 2) [21].

Under the immunostimulatory effect of type I IFN, the cross-presentation capability of DCs and the trafficking and cytotoxicity of $\mathrm{T}$ cells are enhanced by various manners [102]. Firstly, by upregulating the synthesis of MHC and co-stimulatory molecules, as well as CXC Chemokine Receptor 7 (CCR7), type I IFN promotes the maturation and lymph node-homing of DCs $[103,104]$. Secondly, type I IFN stimulates the secretion of Th1 chemokines such as $\mathrm{C}-\mathrm{X}-\mathrm{C}$ motif chemokine ligand 9 (CXCL9) and C-X-C motif chemokine ligand 10 (CXCL10) which further promote the trafficking of $\mathrm{T}$ cells [105]. Thirdly, type I IFN could boost the function of cytotoxic T lymphocytes (CTLs) by increasing the generation of perforin 1 and granzyme B [106]. Moreover, type I IFN stimulates macrophages to secrete various pro-inflammatory cytokines such as interleukin-1 $\beta$ (IL-1 $\beta$ ) [107]. In the meanwhile, type I IFN could weaken the immunosuppressive function of Treg by downregulating the level of cyclic AMP (cAMP) [108]. Generally, DNA damage and cytosolic DNA-mediated cGAS-STING pathway facilitate to reshape an immunosupportive environment. A series of studies demonstrated that PARPi-mediated DNA damage could enhance the recruitment and infiltration of $\mathrm{T}$ cells into tumor via activating cGAS-STING pathway [109-111]. The preexisting TIL is the prerequisite of tumor-killing effect of ICI, thus PARPi could synergize with ICI and decrease the risk of drug resistance [112].

\section{PARPi-mediated PD-L1 upregulation}

The level of PD-L1 is a vital biomarker predicting the efficacy of anti-PD-1/PD-L1 efficacy [86, 113]. The upregulation of PD-L1 is mainly driven by inflammation; thus, the abundance of PD-L1 could reflect the status of tumor immune microenvironment [114]. In cancer cell lines and mouse models, it was observed that PARPi administration induced PD-L1 upregulation [24]. Further exploration identified that the upregulation of PD-L1 was mainly related to the augmented anti-cancer immunity after PARPi treatment [110]. Besides, Sato et al. reported that X-rays or PARPi-induced double-strand breaks could directly upregulate PD-L1 by ATM-ATRChk1 pathway which was independent of IFN pathway [115]. Therefore, additional anti-PD-1/PD-L1 treatment could neutralize the feedback upregulation of PD-L1 and reactivate the blunt tumor-killing activity of TIL. 


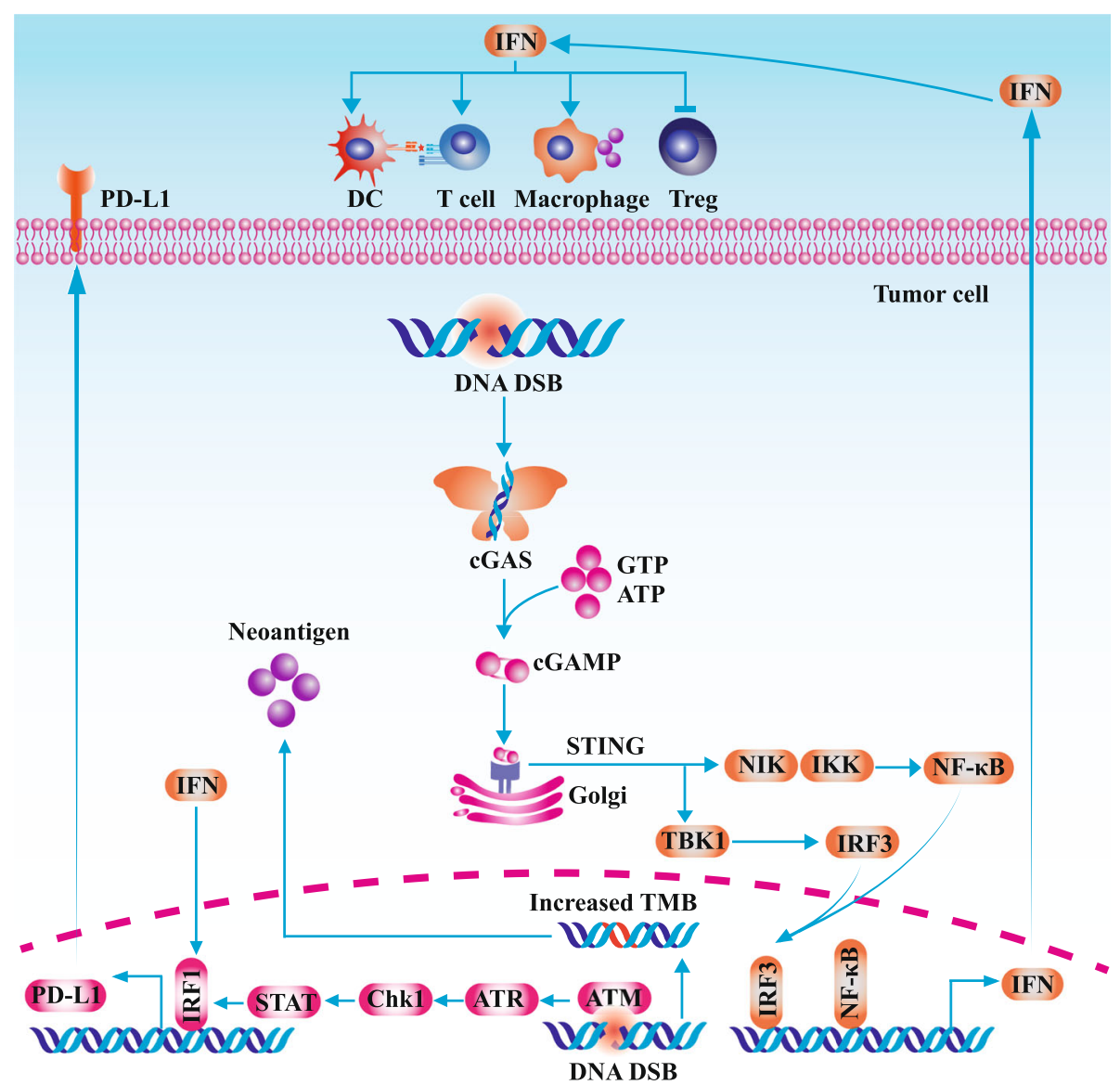

Fig. 2 The cross-talk between DNA damage and immune response. Following the stimulation of cytoplasmic dsDNA, cGAS is activated and catalyzes the generation of cyclic-dinucleotide. CDN is a second messenger which promotes the conformational change of STING. Active STING mainly initiates the downstream TBK1-IRF3-Type I IFN pathway. Besides, STING could activate the NF-KB pathway which cooperates with IRF3 to upregulate the generation of type I IFN. Type I IFN has a substantial influence on systemic immune response and regulates multiple components in anti-cancer immunity. Moreover, PARPi treatment-induced double-strand break could upregulate PD-L1 expression by augmented anti-cancer immunity or ATM-ATR-Chk1 pathway. Lastly, after receiving PARPi treatment, accumulated chromosome rearrangements generate plenty of neoantigens and elevate the immunogenicity of tumor. DSB, double-strand break; STING, stimulator of interferon genes; CGAS cyclic GMP-AMP synthase; TMB, tumor mutation burden

PARPi-mediated reprogram of immune microenvironment The interaction between DDR and immune response is the basis of the combination therapy of PARPi and ICI. In the absence of PARPi, low-level DNA damages at baseline induce the chronic inflammation which promotes the initiation and development of cancers [23]. Actually, the biologic effect of type I IFN is bidirectional which changes along with the timing and magnitude of type I IFN production [116]. Persistent type I IFN secretion at baseline could inhibit the expansion of conventional DCs and upregulates the generation of PD-L1 and IL-10 in DCs and macrophages [116]. PARPi-mediated catastrophic DNA damage and subsequently acute inflammation could rapidly elevate the abundance of type I IFN via cGASSTING pathway [117]. Contrary to the pro-tumor inflammation at baseline, PARPi-mediated acute inflammation remodels tumor immune microenvironment and drives a systemic Th1-skewing immune response [117]. This transformation boosts the priming of immunity and tumorkilling activity, synergizing with ICI for the renaissance of anti-cancer immunity.

\section{The preclinical and clinical studies of PARPi combining $\mathrm{ICI}$ therapy}

Inspired by the synergistic effect of PARPi and ICI treatment, numerous studies are ongoing to explore the actual efficacy of the combination therapy in tumors harboring BRCA $1 / 2$ or other DDR genes mutations (Table 1 ).

\section{PARPi combining with anti-PD-1/PD-L1 treatment}

As early as 2017, Jiao et al. noticed the association between PARP inhibition and treatment-related PD-L1 
Table 1 Ongoing clinical trials exploring the efficacy of PARPi combining ICI treatment

\begin{tabular}{|c|c|c|c|c|}
\hline Intervention & Clinical Trial & Cancer & Phase & Status \\
\hline BGB-A317 and BGB-290 & NCT02660034 & Advanced solid tumors & I & Recruiting \\
\hline Niraparib and Atezolizumab & NCT03598270 & Recurrent ovarian cancer & III & Recruiting \\
\hline Niraparib and PD-1 inhibitor & NCT03308942 & NSCLC & $\|$ & $\begin{array}{l}\text { Active, not } \\
\text { recruiting }\end{array}$ \\
\hline Niraparib and Pembrolizumab & NCT02657889 & TNBC or ovarian cancer & $|/| \mid$ & $\begin{array}{l}\text { Active, not } \\
\text { recruiting }\end{array}$ \\
\hline \multirow[t]{4}{*}{ Niraparib and TSR-042 } & NCT03651206 & Ovarian cancer and endometrial Cancer & $\|/\| \|$ & $\begin{array}{l}\text { Not yet } \\
\text { recruiting }\end{array}$ \\
\hline & NCT03602859 & Stage III or IV non-mEOC & III & Recruiting \\
\hline & NCT03574779 & Recurrent ovarian cancer & $\|$ & Recruiting \\
\hline & NCT03307785 & Advanced or metastatic solid cancer & I & Recruiting \\
\hline Olaparib and Atezolizumab & NCT02849496 & Advanced or metastatic non-HER2-positive breast cancer & $\|$ & Recruiting \\
\hline \multirow[t]{9}{*}{ Olaparib and Durvalumab } & NCT03167619 & TNBC & $\|$ & Recruiting \\
\hline & NCT02546661 & Muscle invasive bladder cancer & I & Recruiting \\
\hline & NCT03459846 & Stage IV platinum-ineligible Urothelial Cancer & $\|$ & Recruiting \\
\hline & NCT03334617 & NSCLC & $\|$ & Recruiting \\
\hline & NCT03851614 & MMR proficient colorectal cancer, pancreatic cancer, and leiomyosarcoma & $\|$ & Recruiting \\
\hline & NCT02734004 & Advanced ovarian, breast, lung, and gastric cancers & $|/| \mid$ & Recruiting \\
\hline & NCT02882308 & Squamous cell carcinoma of the head and neck & $\|$ & Recruiting \\
\hline & NCT03772561 & Advanced solid tumors & I & Recruiting \\
\hline & NCT02484404 & Recurrent ovarian, TNBC, lung, prostate, and colon cancers & $|/| \mid$ & Recruiting \\
\hline \multirow[t]{2}{*}{ Olaparib and Pembrolizumab } & NCT03834519 & mCRPC & III & $\begin{array}{l}\text { Not yet } \\
\text { recruiting }\end{array}$ \\
\hline & NCT02861573 & mCRPC & । & Recruiting \\
\hline Olaparib and Tremelimumab & NCT02571725 & BRCA deficient Ovarian Cancer & $|/| \mid$ & Recruiting \\
\hline $\begin{array}{l}\text { Olaparib, Durvalumab, and } \\
\text { Tremelimumab }\end{array}$ & NCT02953457 & $\begin{array}{l}\text { Recurrent or refractory ovarian, fallopian tube or primary peritoneal } \\
\text { cancer with BRCA mutation }\end{array}$ & $\|$ & Recruiting \\
\hline \multirow[t]{2}{*}{ Rucaparib and Atezolizumab } & NCT03101280 & Advanced gynecologic cancers and TNBC & I & Recruiting \\
\hline & NCT03694262 & Recurrent or progressive endometrial carcinoma. & $\|$ & $\begin{array}{l}\text { Not yet } \\
\text { recruiting }\end{array}$ \\
\hline \multirow[t]{6}{*}{ Rucaparib and Nivolumab } & NCT03639935 & $A B C$ & $\|$ & Recruiting \\
\hline & NCT03572478 & Prostate cancer or endometrial cancer & $|/| \mid$ & Recruiting \\
\hline & NCT03824704 & Selected solid tumors* & $\|$ & $\begin{array}{l}\text { Not yet } \\
\text { recruiting }\end{array}$ \\
\hline & NCT03522246 & Ovarian cancer & III & Recruiting \\
\hline & NCT03338790 & mCRPC & $\|$ & Recruiting \\
\hline & NCT02873962 & Relapsed ovarian, fallopian tube or peritoneal cancer & $\|$ & Recruiting \\
\hline SHR-1210 and SHR3162 & NCT03182673 & Advanced solid tumors & I & Recruiting \\
\hline \multirow[t]{3}{*}{ Talazoparib and Avelumab } & NCT03637491 & Advanced or metastatic RAS-mutant solid tumors & $\|$ & Recruiting \\
\hline & NCT03565991 & BRCA or ATM mutant tumors & $\|$ & Recruiting \\
\hline & NCT03330405 & Advanced or metastatic solid tumors & $\|$ & Recruiting \\
\hline
\end{tabular}

Note: $A B C$ advanced or metastatic biliary tract cancer, $m C R P C$ metastatic castration-resistant prostate cancer, $m E O C$ mucinous epithelial ovarian cancer, NSCLC non-small cell lung cancer, $M M R$ mismatch repair, $T N B C$ triple-negative breast cancer *Including epithelial ovarian cancer, fallopian tube cancer, primary peritoneal carcinoma, metastatic transitional cell cancer of the renal pelvis and ureter, urothelial carcinoma, high-grade serous carcinoma, endometrioid cdenocarcinoma, etc

upregulation [24]. In breast cancer cell lines and xenograft models, PARPi treatment significantly increased the expression of PD-L1 [24]. The results of the co- culture experiment showed that breast cancer cells undergoing Olaparib treatment were resistant to cellkilling activity of activated human peripheral blood 
mononuclear cells [24]. To further investigate whether additional anti-PD-L1 blockade could overcome PARPiinduced immune suppress in vivo, EMT6 syngeneic mouse models were adopted and received anti-PD-L1 blockade/Olaparib monotherapy or combination therapy [24]. Combination therapy exhibited more potent anticancer effect and elevated the abundance of TILs compared with monotherapies [24]. In this study, PARPiinduced PD-L1 upregulation was independent of cGASSTING-IFN pathway [24].

\section{Combination therapy in BRCA1/2 mutated models}

Contrary to the observation of Jiao and colleagues, Ding et al. found PARPi treatment activated STING pathway and triggered robust anti-cancer immunity, as well as induced inflammation-mediated PD-L1 upregulation [118]. Researchers designed two genetically engineered mouse models bearing high-grade serous ovarian cancer: PBM (driven by p53 depletion, BRCA1 depletion, and cMyc overexpression) and PPM (driven by p53 depletion, PTEN depletion, and c-Myc overexpression) [118]. AntiPD-1 monotherapy showed nonsignificant effect on PBM, while concurrent Olaparib combining with antiPD-1 treatment significantly retarded tumor growth [118]. Compared with Olaparib monotherapy, mice receiving combination therapy had prolonged survival time [118]. Further exploration in tumor immune microenvironment revealed that the abundance of TIL increased, the expression of negative co-stimulatory molecules (PD-1/Lag-3/Tim-3) decreased, and the secretion of proinflammation cytokines (IFN- $\gamma$ and TNF- $\alpha$ ) elevated after Olaparib administration [118]. Besides, the expression of CD80/86 and MHC was upregulated on DCs following Olaparib treatment [118]. In the peripheral blood of mice undergoing Olaparib treatment, $\mathrm{CD}^{+} \mathrm{T}$ cells possessed greater capability to produce IFN- $\gamma$ and TNF$\alpha$ [118]. PARPi-mediated local and systemic immune response could be abrogated by STING pathway blockade and enhanced by PD-1 inhibitor [118].

\section{Combination therapy in BRCA1/2 proficient models}

The investigations of combination therapy were mainly conducted in BRCA1/2 mutated tumors [119]. However, it is still controversial that patients without mutations in BRCA or other HR genes could benefit from PARPi combining ICI treatment. Ding et al. found that the combination therapy showed non-significant effect on BRCAproficient ovarian cancers while Wang et al. found the concurrent ICI treatment remarkably enhanced the efficacy of PARPi in multiple BRCA-proficient tumors [120]. Niraparib combined with anti-PD-1/PD-L1 therapy increased the infiltration of immune cells into tumor bed and slowed the tumor growth in BRCA-proficient breast cancer, sarcoma, lung squamous cell carcinoma, and colon adenocarcinoma, as well as bladder cancer [120]. This combination strategy might conduce to broaden the application of PARPi.

Regardless of BRCA status, Sen et al. interrogated the efficacy of PARPi combining with ICI treatment in small cell lung cancer (SCLC) model [110]. SCLC is a unique cancer which is characterized by TP53 and RB loss, as well as MYC amplification [121]. Dysregulated cell cycle checkpoint leads to increased replication stress [122]. In the meanwhile, the loss of RB in SCLC reduces the transcription inhibition of PARP [92]. The viability of SCLC is highly dependent on hyperactive PARP, thus SCLC is prone to be sensitive to PARPi treatment [92]. By activating the STING pathway, the combination therapy of Olaparib and anti-PD-L1 significantly elevated the abundance of $\mathrm{CD}^{+} \mathrm{T}$ cells and $\mathrm{CD}^{+}$cytotoxic $\mathrm{T}$ cells in tumor bed while decreased the infiltration of $\mathrm{PD}-1^{+} / \mathrm{Tim}-3^{+}$exhausted $\mathrm{T}$ cells and $\mathrm{CD}^{2} 5^{+} / \mathrm{FoxP}^{+}{ }^{+}$Tregs [110]. Besides, it was detected that chemokines such as CXCL10 and CCL5 increased after the combination therapy [110]. Although neither Olaparib nor anti-PD-L1 monotherapy could retard tumor growth, the combination therapy induced complete tumor regression and sustained a durable anti-cancer effect in all treated mice [110].

\section{Combination therapy in ongoing clinical trials}

Based on the encouraging results of multiple preclinical studies, a series of clinical trials are ongoing to evaluate the efficacy of PARPi combining ICI treatment in a broad range of cancers. The preliminary data of NCT02484404 showed that the combination therapy of Olaparib and Durvalumab effectively reduced tumor burden (measured by PSA reduction >50\%) in 8/17 unselected metastatic castrate-resistant prostate cancer [123]. Mutation in DDR was a favorable biomarker indicating better response to the combination therapy (12-month progression-free survival probability of deficient DDR vs. proficient DDR, $83.3 \%$ vs. $36.4 \%, P=0.03)[123,124]$. Besides, the results of SCLC cohort of NCT02484404 indicated the baseline TIL status also affected the efficacy of combination strategy [125].

Another clinical study (phase II NCT02734004) explored the effect of Olaparib and Durvalumab combination scheme in germline BRCA-mutated platinum-sensitive relapsed ovarian cancer patients [126]. The interim results indicated this combination strategy was well tolerated. In the meanwhile, the disease control rate (DCR) at 12 weeks was $81 \%$ and the objective response rate (ORR) was $63 \%$ [126]. These early data strongly support the feasibility of the combination scheme containing Olaparib and Durvalumab.

Apart from Olaparib plus Durvalumab strategy, a phase II study NCT02657889 evaluated the effect of Niraparib combining with Pembrolizumab therapy in metastatic triple-negative breast cancer and recurrent ovarian cancer 
patients $[127,128]$. Compared with the therapeutic response in overall enrolled patients, ORR was markedly higher in BRCA1/2 mutated patients (ORR of BRCA1/2 mutated vs. overall patients in breast cancer cohort, $67 \%$ vs. 29\%; ORR of BRCA1/2 mutated vs. overall patients in ovarian cancer cohort, $45 \%$ vs. $25 \%$ ) [127, 128].

\section{PARPi combining with anti-CTLA-4 treatment}

Compared with the intensive attention to anti-PD-1/PDL1, combination scheme of PARPi and anti-CTLA-4 was rarely studied. In 2015, Higuchi et al. conducted a preclinical study to explore the efficacy of PARPi combining with anti-CTLA-4 treatment in BRCA1 deficient ovarian cancer model [129]. In vitro experiment, researchers found that PARPi-induced apoptosis increased when tumor cells were exposed to additional IFN- $\gamma$ or TNF- $\alpha$ [129]. In vivo experiment, anti-CTLA-4 combining with PARPi treatment significantly increased the proportion of effector/ memory $\mathrm{CD}^{+} \mathrm{T}$ cells in tumor microenvironment [129]. Further investigation showed this combination therapy remarkably upregulated the generation of cytokines in TILs [129]. On the contrary, neither anti-CTLA-4 nor PARPi monotherapy could significantly change the abundance and function of lymphocytes [129]. Compared with monotherapy, the combination treatment completely eliminated visible tumor mass and maintained a long-term tumorfree survival in most of mice [129]. The anti-tumor effect of combination therapy was impaired by anti-IFN- $\gamma$ neutralizing antibody [129]. Subsequently adoptive immune cell transfer experiment confirmed the synergistic effect of combination therapy was highly dependent on $\mathrm{T}$ cellmediated immune response [129]. Recipient mice receiving $\mathrm{CD}^{+}$splenocytes from donor mice undergoing combination treatment exhibited the resistance to the following tumor challenge and survived longer than the untreated control group [129]. Combination therapymediated protective immune memory contributed to a durable anti-tumor effect [129].

\section{Conclusion}

DDR deficiency is the driving factor and an essential component of carcinogenesis. As the Achilles' heel of cancer cells, DDR deficiency is an ideal treatment target to interfere genome stability and induce tumor cell death. Based on the synthetic lethal effect, PARPi was initially designed for BRCA deficient patients. Then, it was revealed that PARP inhibition and entrapment induced cytosolic dsDNA formation and subsequent cGAS-STING pathway activation. The cross-talk between PARPi and immune response is the fundament of the combination therapy of PARPi and ICI. Preclinical results and early data of ongoing clinical trials indicated the synergistic effect of PARPi and ICI treatment. By the combination scheme with concurrent ICI, the application of PARPi might be extended to a broad range of cancers far beyond BRCA deficient phenotype. In the meanwhile, PARPi could substantially modulate anti-cancer immune response, enhance immune priming, and reinforce the tumor-killing activity. We believe PARPi is a potential sensitizer for ICI treatment and this novel combination is meaningful for cancer immunotherapy in the future.

\section{Abbreviations}

BER: Base excision repair; CAMP: Cyclic AMP; CCR7: CXC Chemokine Receptor 7; CDN: Cyclic-dinucleotide; CGAS: Cyclic GMP-AMP synthase; CTL: Cytotoxic T lymphocyte; CTLA-4: Cytotoxic T-lymphocyte-associated protein 4;

CXCL10: C-X-C motif chemokine ligand 10; CXCL9: C-X-C motif chemokine ligand 9; DC: Dendritic cell; DCR: Disease control rate; DDR: DNA damage response; DSB: Double-strand break repair; dsDNA: Double-strand DNA;

HR: Homologous recombination; ICl: Immune checkpoint inhibitor; IL-1 $\beta$ : Interleukin-1 $\beta$; ITIM: Immunoreceptor tyrosine-based inhibitory motif; ITSM: Immunoreceptor tyrosine-based switch motif; MHC: Major histocompatibility complex; MMR: Mismatch repair; NER: Nucleotide excision repair; NHEJ: Nonhomologous end joining; NK: Natural killer; ORR: Objective response rate; PARP: Poly ADP-ribose polymerase; PARPi: PARP inhibitor; PD-1: Programmed cell death-1; pMHC: Peptide-major histocompatibility complex; SCLC: Small cell lung cancer; SSB: Single-strand break;

STING: Stimulator of interferon genes; TAA: Tumor-associated antigens; TCR: T cell receptor; TIL: Tumor-infiltrating lymphocyte; Treg: Regulatory T cell

\section{Acknowledgements}

We thank Dr. Tianye Li of the Department of Obstetrics and Gynecology of Tongji Hospital for helpful discussion and language editing assistance.

\section{Authors' contributions \\ $\mathrm{AL}$ and YM performed the selection of literature, drafted the manuscript, and prepared the figures. SQ and QC collected the related references and participated in discussion. KW and SL designed this review and revised the manuscript. All authors contributed to this manuscript. All authors read and approved the final manuscript.}

\section{Funding}

This work was supported by the National Natural Science Foundation of China (No. 81874120, 81572608, 81672984), Wuhan Science and Technology Bureau (No. 2017060201010170).

\section{Availability of data and materials}

Data sharing not applicable to this article as no datasets were generated or analyzed during the current study.

Ethics approval and consent to participate

Not applicable

\section{Consent for publication}

Not applicable

\section{Competing interests}

The authors declare that they have no competing interests.

\section{Author details \\ 'Department of Medical Oncology, The Affiliated Cancer Hospital of Zhengzhou University \& Henan Cancer Hospital, Zhengzhou 450008, China. ${ }^{2}$ Department of Oncology, Tongji Hospital of Tongji Medical College, Huazhong University of Science and Technology, Wuhan 430030, China.}

Received: 3 July 2019 Accepted: 29 August 2019

Published online: 14 September 2019

\section{References}

1. Lee CH, Yelensky R, Jooss K, Chan TA. Update on tumor neoantigens and their utility: why it is good to be different. Trends Immunol. 2018;39:536-48. 2. Li L, Goedegebuure SP, Gillanders WE. Preclinical and clinical development of neoantigen vaccines. Ann Oncol. 2017;28:xii11-xii7. 
3. Yi M, Qin S, Zhao W, Yu S, Chu Q, Wu K. The role of neoantigen in immune checkpoint blockade therapy. Exp Hematol Oncol. 2018;7:28.

4. Wagner S, Mullins CS, Linnebacher M. Colorectal cancer vaccines: Tumor-associated antigens vs neoantigens. World J Gastroenterol. 2018;24:5418-32.

5. Keskin DB, Anandappa AJ, Sun J, Tirosh I, Mathewson ND, Li S, et al. Neoantigen vaccine generates intratumoral T cell responses in phase $\mathrm{lb}$ glioblastoma trial. Nature. 2019;565:234-9.

6. Chen DS, Mellman I. Oncology meets immunology: the cancer-immunity cycle. Immunity. 2013;39:1-10.

7. Marin-Acevedo JA, Soyano AE, Dholaria B, Knutson KL, Lou Y. Cancer immunotherapy beyond immune checkpoint inhibitors. J Hematol Oncol. 2018;11:8.

8. Chen DS, Mellman I. Elements of cancer immunity and the cancer-immune set point. Nature. 2017;541:321-30.

9. Long J, Lin J, Wang A, Wu L, Zheng Y, Yang X, et al. PD-1/PD-L blockade in gastrointestinal cancers: lessons learned and the road toward precision immunotherapy. J Hematol Oncol. 2017;10:146.

10. Bhatia A, Kumar Y. Cellular and molecular mechanisms in cancer immune escape: a comprehensive review. Expert Rev Clin Immunol. 2014;10:41-62.

11. Marin-Acevedo JA, Dholaria B, Soyano AE, Knutson KL, Chumsri S, Lou Y. Next generation of immune checkpoint therapy in cancer: new developments and challenges. J Hematol Oncol. 2018;11:39.

12. Mikkilineni L, Kochenderfer JN. Chimeric antigen receptor T-cell therapies for multiple myeloma. Blood. 2017;130:2594-602.

13. Rowshanravan B, Halliday N, Sansom DM. CTLA-4: a moving target in immunotherapy. Blood. 2018;131:58-67.

14. Yu S, Li A, Liu Q, Li T, Yuan X, Han X, et al. Chimeric antigen receptor T cells: a novel therapy for solid tumors. J Hematol Oncol. 2017;10:78.

15. Horn L, Mansfield AS, Szczesna A, Havel L, Krzakowski M, Hochmair MJ, et al. First-line atezolizumab plus chemotherapy in extensive-stage small-cell lung cancer. N Engl J Med. 2018;379:2220-9.

16. Berzofsky JA, Terabe M, Trepel JB, Pastan I, Stroncek DF, Morris JC, et al. Cancer vaccine strategies: translation from mice to human clinical trials. Cancer Immunol Immunother. 2018;67:1863-9.

17. Yi M, Jiao D, Xu H, Liu Q, Zhao W, Han X, et al. Biomarkers for predicting efficacy of PD-1/PD-L1 inhibitors. Mol Cancer. 2018;17:129.

18. Wang Z, Wu Z, Liu Y, Han W. New development in CAR-T cell therapy. J Hematol Oncol. 2017:10:53.

19. Liu D, Wang S, Bindeman W. Clinical applications of PD-L1 bioassays for cancer immunotherapy. J Hematol Oncol. 2017:10:110.

20. Yi M, Jiao D, Qin S, Chu Q, Wu K, Li A. Synergistic effect of immune checkpoint blockade and anti-angiogenesis in cancer treatment. Mol Cancer. 2019;18:60

21. Li A, Yi M, Qin S, Song Y, Chu Q, Wu K. Activating CGAS-STING pathway for the optimal effect of cancer immunotherapy. J Hematol Oncol. 2019;12:35.

22. Eustermann S, Wu WF, Langelier MF, Yang JC, Easton LE, Riccio AA, et al. Structural basis of detection and signaling of DNA single-strand breaks by human PARP-1. Mol Cell. 2015:60:742-54.

23. Stewart RA, Pilie PG, Yap TA. Development of PARP and immune-checkpoint inhibitor combinations. Cancer Res. 2018;78:6717-25.

24. Jiao S, Xia W, Yamaguchi H, Wei Y, Chen MK, Hsu JM, et al. PARP inhibitor upregulates PD-L1 expression and enhances cancer-associated immunosuppression. Clin Cancer Res. 2017;23:3711-20.

25. Sirbu BM, Cortez D. DNA damage response: three levels of DNA repair regulation. Cold Spring Harb Perspect Biol. 2013;5:a012724.

26. Jackson SP, Bartek J. The DNA-damage response in human biology and disease. Nature. 2009;461:1071-8

27. Brown JS, O'Carrigan B, Jackson SP, Yap TA. Targeting DNA repair in cancer: beyond PARP inhibitors. Cancer Discov. 2017;7:20-37.

28. Tubbs A, Nussenzweig A. Endogenous DNA damage as a source of genomic instability in cancer. Cell. 2017;168:644-56.

29. Lord CJ, Ashworth A. PARP inhibitors: synthetic lethality in the clinic. Science. 2017:355:1152-8.

30. De Vos M, Schreiber $V$, Dantzer $F$. The diverse roles and clinical relevance of PARPs in DNA damage repair: current state of the art. Biochem Pharmacol. 2012;84:137-46.

31. Liu L, Kong M, Gassman NR, Freudenthal BD, Prasad R, Zhen S, et al. PARP1 changes from three-dimensional DNA damage searching to onedimensional diffusion after auto-PARylation or in the presence of APE1. Nucleic Acids Res. 2017;45:12834-47.
32. Wang YQ, Wang PY, Wang YT, Yang GF, Zhang A, Miao ZH. An update on Poly(ADP-ribose)polymerase-1 (PARP-1) inhibitors: opportunities and challenges in cancer therapy. J Med Chem. 2016;59:9575-98.

33. Pettitt SJ, Lord CJ. Dissecting PARP inhibitor resistance with functional genomics. Curr Opin Genet Dev. 2019;54:55-63.

34. Farmer $\mathrm{H}, \mathrm{McC}$ abe $\mathrm{N}$, Lord CJ, Tutt AN, Johnson DA, Richardson TB, et al. Targeting the DNA repair defect in BRCA mutant cells as a therapeutic strategy. Nature. 2005;434:917-21.

35. Bergs JW, Krawczyk PM, Borovski T, ten Cate R, Rodermond HM, Stap J, et al. Inhibition of homologous recombination by hyperthermia shunts early double strand break repair to non-homologous end-joining. DNA Repair (Amst). 2013;12:38-45

36. Agarwal S, Tafel AA, Kanaar R. DNA double-strand break repair and chromosome translocations. DNA Repair (Amst). 2006;5:1075-81.

37. Faraoni I, Graziani G. Role of BRCA mutations in cancer treatment with Poly(ADP-ribose) polymerase (PARP) inhibitors. Cancers (Basel). 2018. https:// doi.org/10.3390/cancers10120487.

38. Povirk LF. Biochemical mechanisms of chromosomal translocations resulting from DNA double-strand breaks. DNA Repair (Amst). 2006;5:1199-212.

39. Lowery MA, Kelsen DP, Capanu M, Smith SC, Lee JW, Stadler ZK, et al. Phase II trial of veliparib in patients with previously treated BRCA-mutated pancreas ductal adenocarcinoma. Eur J Cancer. 2018;89:19-26.

40. Swisher EM, Lin KK, Oza AM, Scott CL, Giordano H, Sun J, et al. Rucaparib in relapsed, platinum-sensitive high-grade ovarian carcinoma (ARIEL2 Part 1): an international, multicentre, open-label, phase 2 trial. Lancet Oncol. 2017;18:75-87.

41. Robson M, Im SA, Senkus E, Xu B, Domchek SM, Masuda N, et al. Olaparib for metastatic breast cancer in patients with a germline BRCA mutation. N Engl J Med. 2017;377:523-33.

42. Pujade-Lauraine E, Ledermann JA, Selle F, Gebski V, Penson RT, Oza AM, et al. Olaparib tablets as maintenance therapy in patients with platinum-sensitive, relapsed ovarian cancer and a BRCA1/2 mutation (SOLO2/ENGOT-OV21): a double-blind, randomised, placebo-controlled phase 3 trial. Lancet Oncol. 2017:18:1274-84.

43. Mirza MR, Monk BJ, Herrstedt J, Oza AM, Mahner S, Redondo A, et al. Niraparib maintenance therapy in platinum-sensitive, recurrent ovarian cancer. N Engl J Med. 2016:375:2154-64.

44. Turner NC, Telli ML, Rugo HS, Mailliez A, Ettl J, Grischke EM, et al. A phase II study of talazoparib after platinum or cytotoxic nonplatinum regimens in patients with advanced breast cancer and germline BRCA1/2 mutations (ABRAZO). Clin Cancer Res. 2019:25:2717-24.

45. Murai J, Huang SY, Das BB, Renaud A, Zhang Y, Doroshow JH, et al. Trapping of PARP1 and PARP2 by clinical PARP inhibitors. Cancer Res. 2012;72:5588-99.

46. Helleday T. The underlying mechanism for the PARP and BRCA synthetic lethality: clearing up the misunderstandings. Mol Oncol. 2011;5:387-93.

47. Franzese E, Centonze S, Diana A, Carlino F, Guerrera LP, Di Napoli M, et al. PARP inhibitors in ovarian cancer. Cancer Treat Rev. 2019;73:1-9.

48. Dulaney C, Marcrom S, Stanley J, Yang ES. Poly(ADP-ribose) polymerase activity and inhibition in cancer. Semin Cell Dev Biol. 2017;63:144-53.

49. Hu X, Huang W, Fan M. Emerging therapies for breast cancer. J Hematol Oncol. 2017; 10:98

50. Lord CJ, Ashworth A. BRCAness revisited. Nat Rev Cancer. 2016:16:110-20.

51. Lord CJ, Ashworth A. Mechanisms of resistance to therapies targeting BRCA-mutant cancers. Nat Med. 2013:19:1381-8.

52. Jaspers JE, Kersbergen A, Boon U, Sol W, van Deemter L, Zander SA, et al. Loss of 53BP1 causes PARP inhibitor resistance in Brca1-mutated mouse mammary tumors. Cancer Discov. 2013;3:68-81.

53. Pettitt SJ, Rehman FL, Bajrami I, Brough R, Wallberg F, Kozarewa I, et al. A genetic screen using the PiggyBac transposon in haploid cells identifies Parp1 as a mediator of olaparib toxicity. PLoS One. 2013;8:e61520.

54. Barber LJ, Sandhu S, Chen L, Campbell J, Kozarewa I, Fenwick K, et al. Secondary mutations in BRCA2 associated with clinical resistance to a PARP inhibitor. J Pathol. 2013:229:422-9.

55. Dhawan MS, Bartelink IH, Aggarwal RR, Leng J, Zhang JZ, Pawlowska N, et al. Differential toxicity in patients with and without DNA repair mutations: phase I study of carboplatin and talazoparib in advanced solid tumors. Clin Cancer Res. 2017:23:6400-10.

56. Esteva FJ, Hubbard-Lucey VM, Tang J, Pusztai L. Immunotherapy and targeted therapy combinations in metastatic breast cancer. Lancet Oncol. 2019;20:e175-e86. 
57. Moore K, Colombo N, Scambia G, Kim BG, Oaknin A, Friedlander M, et al. Maintenance olaparib in patients with newly diagnosed advanced ovarian cancer. N Engl J Med. 2018;379:2495-505.

58. Golan T, Hammel P, Reni M, Van Cutsem E, Macarulla T, Hall MJ, et al. Maintenance olaparib for germline BRCA-mutated metastatic pancreatic cancer. N Engl J Med. 2019. https://doi.org/10.1056/NEJMoa1903387.

59. Bretscher PA. A two-step, two-signal model for the primary activation of precursor helper T cells. Proc Natl Acad Sci U S A. 1999;96:185-90.

60. Appleman $L$, Boussiotis VA. T cell anergy and costimulation. Immunol Rev. 2003;192:161-80.

61. Oosterwegel MA, Greenwald RJ, Mandelbrot DA, Lorsbach RB, Sharpe AH. CTLA-4 and T cell activation. Curr Opin Immunol. 1999;11:294-300.

62. Salomon B, Bluestone JA. Complexities of CD28/B7: CTLA-4 costimulatory pathways in autoimmunity and transplantation. Annu Rev Immunol. 2001;19:225-52.

63. Yamazaki T, Akiba H, Iwai $H$, Matsuda $H$, Aoki M, Tanno $Y$, et al. Expression of programmed death 1 ligands by murine T cells and APC. J Immunol. 2002;169:5538-45.

64. Keir ME, Butte MJ, Freeman GJ, Sharpe AH. PD-1 and its ligands in tolerance and immunity. Annu Rev Immunol. 2008;26:677-704.

65. Bardhan K, Anagnostou T, Boussiotis VA. The PD1:PD-L1/2 pathway from discovery to clinical implementation. Front Immunol. 2016;7:550.

66. Boussiotis VA, Chatterjee P, Li L. Biochemical signaling of PD-1 on T cells and its functional implications. Cancer J. 2014;20:265-71.

67. Sheppard KA, Fitz $\sqcup$, Lee JM, Benander C, George JA, Wooters J, et al. PD-1 inhibits T-cell receptor induced phosphorylation of the ZAP70/CD3zeta signalosome and downstream signaling to PKCtheta. FEBS Lett. 2004;574:37-41.

68. Patsoukis N, Brown J, Petkova V, Liu F, Li L, Boussiotis VA. Selective effects of PD-1 on Akt and Ras pathways regulate molecular components of the cell cycle and inhibit T cell proliferation. Sci Signal. 2012;5:ra46.

69. Parry RV, Chemnitz JM, Frauwirth KA, Lanfranco AR, Braunstein I, Kobayashi SV, et al. CTLA-4 and PD-1 receptors inhibit T-cell activation by distinct mechanisms. Mol Cell Biol. 2005;25:9543-53.

70. Chang $\mathrm{CH}$, Curtis JD, Maggi LB Jr, Faubert B, Villarino AV, O'Sullivan D, et al. Posttranscriptional control of $T$ cell effector function by aerobic glycolysis. Cell. 2013;153:1239-51

71. Wilson RAM, Evans TRJ, Fraser AR, Nibbs RJB. Immune checkpoint inhibitors: new strategies to checkmate cancer. Clin Exp Immunol. 2018;191:133-48.

72. Ok CY, Young KH. Checkpoint inhibitors in hematological malignancies. J Hematol Oncol. 2017:10:103.

73. Soskic B, Qureshi OS, Hou T, Sansom DM. A transendocytosis perspective on the CD28/CTLA-4 pathway. Adv Immunol. 2014;124:95-136.

74. Rudd CE, Taylor A, Schneider H. CD28 and CTLA-4 coreceptor expression and signal transduction. Immunol Rev. 2009;229:12-26.

75. Robert C, Thomas L, Bondarenko I, O'Day S, Weber J, Garbe C, et al. Ipilimumab plus dacarbazine for previously untreated metastatic melanoma. N Engl J Med. 2011;364:2517-26.

76. Mehta K, Patel K, Parikh RA. Immunotherapy in genitourinary malignancies. J Hematol Oncol. 2017:10:95

77. Nanda R, Chow LQ, Dees EC, Berger R, Gupta S, Geva R, et al. Pembrolizumab in patients with advanced triple-negative breast cancer: Phase Ib KEYNOTE-012 Study. J Clin Oncol. 2016;34:2460-7.

78. Forde PM, Chaft JE, Smith KN, Anagnostou V, Cottrell TR, Hellmann MD, et al. Neoadjuvant PD-1 blockade in resectable lung cancer. N Engl J Med. 2018:378:1976-86

79. Atkins MB, Plimack ER, Puzanov I, Fishman MN, McDermott DF, Cho DC, et al. Axitinib in combination with pembrolizumab in patients with advanced renal cell cancer: a non-randomised, open-label, dose-finding, and dose-expansion phase 1b trial. Lancet Oncol. 2018;19:405-15.

80. Eggermont AMM, Blank CU, Mandala M, Long GV, Atkinson V, Dalle S, et al. Adjuvant pembrolizumab versus placebo in resected stage III melanoma. N Engl J Med. 2018;378:1789-801.

81. Massard C, Gordon MS, Sharma S, Rafii S, Wainberg ZA, Luke J, et al. Safety and efficacy of durvalumab (MED|4736), an anti-programmed cell death Ligand-1 immune checkpoint inhibitor, in patients with advanced urothelial bladder cancer. J Clin Oncol. 2016:34:3119-25.

82. Xu-Monette ZY, Zhang M, Li J, Young KH. PD-1/PD-L1 Blockade: have we found the key to unleash the antitumor immune response? Front Immunol. 2017:8:1597

83. Yarchoan $\mathrm{M}$, Hopkins $\mathrm{A}$, Jaffee EM. Tumor mutational burden and response rate to PD-1 inhibition. N Engl J Med. 2017;377:2500-1.
84. Jin Z, Yoon HH. The promise of PD-1 inhibitors in gastro-esophageal cancers: microsatellite instability vs. PD-L1. J Gastrointest Oncol. 2016;7:771-88.

85. Teng MW, Ngiow SF, Ribas A, Smyth MJ. Classifying cancers based on T-cell infiltration and PD-L1. Cancer Res. 2015;75:2139-45.

86. Reck M, Rodriguez-Abreu D, Robinson AG, Hui R, Csoszi T, Fulop A, et al. Pembrolizumab versus Chemotherapy for PD-L1-positive non-small-cell lung cancer. N Engl J Med. 2016;375:1823-33.

87. Gong X, Li X, Jiang T, Xie H, Zhu Z, Zhou F, et al. Combined radiotherapy and anti-PD-L1 antibody synergistically enhances antitumor effect in non-small cell lung cancer. J Thorac Oncol. 2017;12:1085-97.

88. Dafni U, Tsourti Z, Vervita K, Peters S. Immune checkpoint inhibitors, alone or in combination with chemotherapy, as first-line treatment for advanced non-small cell lung cancer. A systematic review and network meta-analysis. Lung Cancer. 2019:134:127-40.

89. Sullivan RJ, Hamid O, Gonzalez R, Infante JR, Patel MR, Hodi FS, et al. Atezolizumab plus cobimetinib and vemurafenib in BRAF-mutated melanoma patients. Nat Med. 2019;25:929-35.

90. Chowdhury PS, Chamoto K, Honjo T. Combination therapy strategies for improving PD-1 blockade efficacy: a new era in cancer immunotherapy. J Intern Med. 2018;283:110-20.

91. Migden MR, Rischin D, Schmults CD, Guminski A, Hauschild A, Lewis KD, et al. PD-1 blockade with cemiplimab in advanced cutaneous squamous-cell carcinoma. N Engl J Med. 2018;379:341-51.

92. Pilie PG, Gay CM, Byers LA, O'Connor MJ, Yap TA. PARP inhibitors: extending benefit beyond BRCA-mutant cancers. Clin Cancer Res. 2019;25:3759-71.

93. Mehnert JM, Panda A, Zhong H, Hirshfield K, Damare S, Lane K, et al. Immune activation and response to pembrolizumab in POLE-mutant endometrial cancer. J Clin Invest. 2016;126:2334-40.

94. Strickland KC, Howitt BE, Shukla SA, Rodig S, Ritterhouse LL, Liu JF, et al. Association and prognostic significance of BRCA1/2-mutation status with neoantigen load, number of tumor-infiltrating lymphocytes and expression of PD-1/PD-L1 in high grade serous ovarian cancer. Oncotarget. 2016:7:13587-98.

95. Teo MY, Seier K, Ostrovnaya I, Regazzi AM, Kania BE, Moran MM, et al. Alterations in DNA damage response and repair genes as potential marker of clinical benefit from PD-1/PD-L1 blockade in advanced urothelial cancers. J Clin Oncol. 2018;36:1685-94.

96. Mouw KW, Goldberg MS, Konstantinopoulos PA, D'Andrea AD. DNA damage and repair biomarkers of immunotherapy response. Cancer Discov. 2017;7:675-93.

97. Khoo LT, Chen LY. Role of the CGAS-STING pathway in cancer development and oncotherapeutic approaches. EMBO Rep. 2018. https://doi.org/10. 15252/embr.201846935.

98. Gao P, Ascano M, Wu Y, Barchet W, Gaffney BL, Zillinger T, et al. Cyclic $\left[\mathrm{G}\left(2^{\prime}, 5^{\prime}\right) \mathrm{pA}\left(3^{\prime}, 5^{\prime}\right) \mathrm{p}\right]$ is the metazoan second messenger produced by DNAactivated cyclic GMP-AMP synthase. Cell. 2013:153:1094-107.

99. Barber GN. STING: infection, inflammation and cancer. Nat Rev Immunol. 2015;15:760-70.

100. Ishikawa H, Ma Z, Barber GN. STING regulates intracellular DNA-mediated, type I interferon-dependent innate immunity. Nature. 2009;461:788-92.

101. Abe T, Barber GN. Cytosolic-DNA-mediated, STING-dependent proinflammatory gene induction necessitates canonical NF-kappaB activation through TBK1. J Virol. 2014;88:5328-41.

102. Zitvogel L, Galluzzi L, Kepp O, Smyth MJ, Kroemer G. Type I interferons in anticancer immunity. Nat Rev Immunol. 2015;15:405-14.

103. Simmons DP, Wearsch PA, Canaday DH, Meyerson HJ, Liu YC, Wang Y, et al. Type I IFN drives a distinctive dendritic cell maturation phenotype that allows continued class II MHC synthesis and antigen processing. J Immunol. 2012:188:3116-26.

104. Parlato S, Santini SM, Lapenta C, Di Pucchio T, Logozzi M, Spada M, et al. Expression of CCR-7, MIP-3beta, and Th-1 chemokines in type I IFN-induced monocyte-derived dendritic cells: importance for the rapid acquisition of potent migratory and functional activities. Blood. 2001;98:3022-9.

105. Peng D, Kryczek I, Nagarsheth N, Zhao L, Wei S, Wang W, et al. Epigenetic silencing of TH1-type chemokines shapes tumour immunity and immunotherapy. Nature. 2015;527:249-53.

106. Guillot B, Portales P, Thanh AD, Merlet S, Dereure O, Clot J, et al. The expression of cytotoxic mediators is altered in mononuclear cells of patients with melanoma and increased by interferon-alpha treatment. $\mathrm{Br}$ J Dermatol. 2005;152:690-6. 
107. Novikov A, Cardone M, Thompson R, Shenderov K, Kirschman KD, MayerBarber KD, et al. Mycobacterium tuberculosis triggers host type I IFN signaling to regulate IL-1beta production in human macrophages. J Immunol. 2011;187:2540-7.

108. Bacher N, Raker V, Hofmann C, Graulich E, Schwenk M, Baumgrass R, et al. Interferon-alpha suppresses CAMP to disarm human regulatory T cells. Cancer Res. 2013;73:5647-56

109. Pantelidou C, Sonzogni O, de Oliveira TM, Mehta AK, Kothari A, Wang D, et al. PARP inhibitor efficacy depends on CD8+ T cell recruitment via intratumoral STING pathway activation in BRCA-deficient models of triplenegative breast cancer. Cancer Discov. 2019;9:722-37.

110. Sen T, Rodriguez BL, Chen L, Corte CMD, Morikawa N, Fujimoto J, et al. Targeting DNA damage response promotes antitumor immunity through STING-mediated T-cell activation in small cell lung cancer. Cancer Discov. 2019;9:646-61

111. Shen J, Zhao W, Ju Z, Wang L, Peng Y, Labrie M, et al. PARPi Triggers the STING-dependent immune response and enhances the therapeutic efficacy of immune checkpoint blockade independent of BRCAness. Cancer Res. 2019;79:311-9.

112. Drapkin BJ, Farago AF. Unexpected synergy reveals new therapeutic strategy in SCLC. Trends Pharmacol Sci. 2019;40:295-7.

113. Ding W, LaPlant BR, Call TG, Parikh SA, Leis JF, He R, et al. Pembrolizumab in patients with CLL and Richter transformation or with relapsed CLL. Blood. 2017;129:3419-27.

114. Gao Y, Yang J, Cai Y, Fu S, Zhang N, Fu X, et al. IFN-gamma-mediated inhibition of lung cancer correlates with PD-L1 expression and is regulated by PI3K-AKT signaling. Int J Cancer. 2018;143:931-43.

115. Sato H, Niimi A, Yasuhara T, Permata TBM, Hagiwara Y, Isono M, et al. DNA double-strand break repair pathway regulates PD-L1 expression in cancer cells. Nat Commun. 2017:8:1751.

116. Lee AJ, Ashkar AA. The dual nature of Type I and Type II interferons. Front Immunol. 2018;9:2061.

117. Chabanon RM, Muirhead G, Krastev DB, Adam J, Morel D, Garrido M, et al. PARP inhibition enhances tumor cell-intrinsic immunity in ERCC1-deficient non-small cell lung cancer. J Clin Invest. 2019;129:1211-28.

118. Ding $L$, Kim HJ, Wang Q, Kearns $M$, Jiang T, Ohlson CE, et al. PARP unhibition elicits STING-dependent antitumor immunity in Brca1-deficient ovarian cancer. Cell Rep. 2018;25:2972-80.e5.

119. Criscuolo D, Morra F, Giannella R, Visconti R, Cerrato A, Celetti A. New combinatorial strategies to improve the PARP inhibitors efficacy in the urothelial bladder Cancer treatment. J Exp Clin Cancer Res. 2019;38:91.

120. Wang Z, Sun K, Xiao Y, Feng B, Mikule K, Ma X, et al. Niraparib activates interferon signaling and potentiates anti-PD-1 antibody efficacy in tumor models. Sci Rep. 2019;9:1853.

121. George J, Lim JS, Jang SJ, Cun Y, Ozretic L, Kong G, et al. Comprehensive genomic profiles of small cell lung cancer. Nature. 2015;524:47-53.

122. Byers LA, Wang J, Nilsson MB, Fujimoto J, Saintigny P, Yordy J, et al. Proteomic profiling identifies dysregulated pathways in small cell lung cancer and novel therapeutic targets including PARP1. Cancer Discov. 2012;2:798-811.

123. Karzai F, Madan RA, Owens $H$, Couvillon A, Hankin A, Williams M, et al. A phase 2 study of olaparib and durvalumab in metastatic castrate-resistant prostate cancer (mCRPC) in an unselected population. J Clin Oncol. 2018; 36(suppl 6):163.

124. Karzai F, VanderWeele D, Madan RA, Owens H, Cordes LM, Hankin A, et al. Activity of durvalumab plus olaparib in metastatic castration-resistant prostate cancer in men with and without DNA damage repair mutations. J Immunother Cancer. 2018;6:141.

125. Thomas A, Vilimas R, Trindade C, Erwin-Cohen R, Roper N, Xi L, et al. Durvalumab in combination with olaparib in patients with relapsed small cell lung cancer: results from a phase II study. J Thorac Oncol. 2019. https:// doi.org/10.1016/j.jtho.2019.04.026.

126. Drew $Y$, de Jonge $M$, Hong SH, Park $Y H$, Wolfer A, Brown J, et al. An open-label, phase II basket study of olaparib and durvalumab (MEDIOLA): Results in germline BRCA-mutated (gBRCAm) platinum-sensitive relapsed (PSR) ovarian cancer (OC). Gynecol Oncol. 2018;149(suppl 1):246-7.

127. Vinayak S, Tolaney SM, Schwartzberg LS, Mita MM, McCann GA-L, Tan AR, et al. TOPACIO/Keynote-162: Niraparib + pembrolizumab in patients (pts) with metastatic triple-negative breast cancer (TNBC), a phase 2 trial. J Clin Oncol. 2018;36(suppl 15):1011.
128. Konstantinopoulos PA, Waggoner SE, Vidal GA, Mita MM, Fleming GF, Holloway RW, et al. TOPACIO/Keynote-162 (NCT02657889): A phase 1/2 study of niraparib + pembrolizumab in patients (pts) with advanced triple-negative breast cancer or recurrent ovarian cancer (ROC)-results from ROC cohort. J Clin Oncol. 2018;36(suppl 15):106.

129. Higuchi T, Flies DB, Marjon NA, Mantia-Smaldone G, Ronner L, Gimotty PA, et al. CTLA-4 blockade synergizes therapeutically with PARP inhibition in BRCA1-deficient ovarian cancer. Cancer Immunol Res. 2015;3:1257-68.

\section{Publisher's Note}

Springer Nature remains neutral with regard to jurisdictional claims in published maps and institutional affiliations.
Ready to submit your research? Choose BMC and benefit from:

- fast, convenient online submission

- thorough peer review by experienced researchers in your field

- rapid publication on acceptance

- support for research data, including large and complex data types

- gold Open Access which fosters wider collaboration and increased citations

- maximum visibility for your research: over $100 \mathrm{M}$ website views per year

At $\mathrm{BMC}$, research is always in progress.

Learn more biomedcentral.com/submissions 